Conference Secretariat (tel: 01603711 258; fax: 01603711 202; e-mail: julie.allen@nchp-trust.anglox.nhs.uk). CPD points: 6

The International Federation of Psychiatric Epidemiology (IFPE) would like to announce Mental Health Epidemiology and Service Needs, an Asia/Pacific regional conference which will be held in Kuala Lumpur, Malaysia on 2630 September 2001. The purpose of the conference, which will include a workshop in epidemiological methods, is to bring together clinicians and scientists who are engaged in epidemiological or health service research into mental disorders, to review progress especially in the Asian and Pacific regions and to help promote international collaboration in this field. Further details are given on the IFPE home page (http://www.humv.es/uipcs/ifpe). Those interested should contact Professor Saroja Krishnaswamy, Department of Psychiatry, Hospital University Kebangsaan Malaysia Jalan Yaacob Latif, Bandar Tun Razak 56000 Cheras, Kuala Lumpur, Malaysia (tel: 00603787 46634; fax: 00603787 56304/3973 784; e-mail: saroja54@hotmail.com).

IPT In The UK: 2nd National Conference is a one-day conference on interpersonal psychotherapy (IPT) to be held at the Royal College of Physicians in
Edinburgh on 26 September 2000. The conference aims are to provide an update on recent developments in IPT; to allow those with an interest in IPT in the UK/ Europe to network; and to discuss issues of training and accreditation in IPT. Further details and application form from website: www.IPTEdinburgh.il2.com or IPT Edinburgh, The Cottage, Royal Edinburgh Hospital, Morningside, Edinburgh EH10 5HF (tel: 0131537 6838).

The Royal Free \& University College Medical School, Department of Psychiatry and Behavioural Sciences are inviting applications for two courses. Semester One of the MRCPsych Course will take place on Monday afternoons commencing 4 September 2000 (Part I) and Tuesday afternoons commencing 5 September 2000 (Part II). This course includes mock exams, theme specific revision sessions, research methodology and the Royal College's Critical Appraisal Paper for the Part II exam. The MSc in Psychiatric Theory and Research is a two-year, part-time course starting in October 2000. The MSc is intended for senior house officers and specialist registrars in psychiatry and aims to provide a firm grounding in research methodology. In the first year of the course, students will need to attend for two afternoons a week which will involve seminars, tutorials, workshops and lectures. In the second year, students will conduct a research project under supervision and submit a dissertation. Places on the MSc course are limited to 15 students so early application is advisable. Further information about both courses: Linda Dutton, Course Administrator (tel: 0207679 9475; e-mail: I.dutton@ucl.ac.uk).

Coventry Healthcare NHS Trust are the organisers of 'Back to the Future' - A Conference to Celebrate Older Persons Mental Health Services in Recognition of World Mental Health Day. This conference will take place on 1011 October 2000 and will cover the following main themes: National Service Framework, Changing Cultures, Person Centred Standards, Law and Ethics and Alzheimer's Younger Sufferer. Further information: Pat Andrusko, Coventry Healthcare NHS Trust, Clifford Bridge Road, Coventry CV2 2TE (tel./fax: 024 7653 8984).

The Association of Therapeutic Communities (ATC) are the sponsors of Windsor Conference: Creating a Healthy Balance: Nourishing Body, Mind and Soul, an annual international event taking place on 1114 September 2000. Further information: ATC, 1315 Pine Street, London EC1R 0JG (e-mail: post@ therapeuticcommunities.org).

\title{
corrigendum
}

\section{How long can a waiting list be?}

On page 212 of the paper by Foreman \& Hanna (Psychiatric Bulletin, June
2000, 24, 211-213), in the 'Findings' section, the second sentence should read:

"Ten per cent of those returning questionnaires did not attend the appointments they were sent, and a further $\mathbf{1 0 . 8} \%$ did not require an appointment, as no current psychiatric problem could be identified from the questionnaire or letter." 